\title{
The role of carbon nanoparticles in guiding central neck dissection and protecting the parathyroid in transoral vestibular endoscopic thyroidectomy for thyroid cancer
}

\author{
Zhiliang $\mathrm{Xu}^{1}$, Yanyan Meng ${ }^{2}$, Junlong Song ${ }^{1}$, Yuan Wang $^{1}$, Xiaoli Yao ${ }^{1}$ \\ ${ }^{1}$ Department of Breast and Thyroid Surgery, Renmin Hospital of Wuhan University, Wuhan, Hubei, China \\ ${ }^{2}$ Department of Cardiology, Renmin Hospital of Wuhan University, Wuhan, Hubei, China
}

Videosurgery Miniinv 2020; 15 (3): 455-461

DOI: https://doi.org/10.5114/wiitm.2019.89658

\begin{abstract}
Introduction: Transoral vestibular endoscopic thyroidectomy (TOET) is sometimes used in young and middle-aged patients with papillary thyroid microcarcinoma (PTMC), but it is still difficult to identify lymph nodes (LNS) and parathyroid glands (PGs). Carbon nanoparticle (CN) is a novel lymph node tracer and has been widely used in open thyroid surgery. Aim: To evaluate the efficacy of CN in identifying LNs and preserving PGs in TOET with central neck dissection (CND). Material and methods: A total of 72 PTMC patients undergoing TOET with CND were retrospectively enrolled from January 2017 to January 2019. Patients were divided into a CN group $(n=38)$ and a control group $(n=34)$. The parameters including pathological characteristics, surgery related indicators, serum $\mathrm{Ca}^{2+}$ and parathyroid hormone (PTH) levels were compared.

Results: No significant differences were found in patient characteristics, operative complications and superior PGs preserved in situ (all $p>0.05$ ). Total LNs and number of LNs less than $5 \mathrm{~mm}$ were significantly higher in the CN group than in the control group $(p=0.021, p<0.01)$. The number of superior $P G s$ preserved in situ discovered in the $C N$ group was greater than the control group $(p=0.038)$. Serum PTH and $\mathrm{Ca}^{2+}$ levels dropped markedly in each group after surgery and gradually recovered in time. The $C N$ group recovered faster than the control group.

Conclusions: CN may be a good choice for TOET for PTMC because of better protection and faster recovery of parathyroid function, and more LNs removed.
\end{abstract}

Key words: parathyroid, endoscopic thyroidectomy, carbon nanoparticle, central lymph node, transoral vestibular.

\section{Introduction}

Central lymph node metastasis sometimes occurs in papillary thyroid microcarcinoma (PTMC) in young and middle-aged women [1-4]. These patients are often concerned about the cosmetic appearance of the surgical scar, and this concern has led to increased interest in transoral vestibular endoscopic thyroidectomy (TOET) among clinicians and patients [5-7]. TOET offers a magnified operative field, higher resolution vision, and clearer exposure of the operation site when compared to traditional open surgery, but identification of lymph nodes and parathyroid glands (PGs) is still difficult [8-10]. Carbon nanoparticles (CNs) are novel lymph node tracers that have been widely used in open thyroid surgery [11, 12]. The goal of this study was to retrospectively evaluate the use of CNs in TOET in patients with unilateral PTMC. 


\section{Aim}

To evaluate the efficacy of CNs for identifying lymph nodes (LNs) and preserving parathyroid glands in TOET with central neck dissection (CND).

\section{Material and methods}

A retrospective review was performed using the records of 72 consecutive PTMC patients who underwent transoral vestibular unilateral thyroidectomy with CND in the Breast and Thyroid Surgery Department of Wuhan University from January 2017 to January 2019. Patients were preoperatively diagnosed with PTMC based on ultrasound guided fine needle aspiration cytology and were retrospectively divided into two groups: a $\mathrm{CN}$ group $(n=38)$ and a control group $(n=34)$. Patients were excluded if they met one or more of the following criteria: (1) contraindications for anesthesia, such as pregnancy, multiple organ failure, or mental disease; (2) history of neck surgery or radiotherapy; (3) concomitant Graves' disease or a huge benign nodule; (4) evidence of local invasion or involvement of the recurrent laryngeal nerve (RLN); (5) preoperative imaging revealing enlarged and fused LNs; and (6) preoperative hypoparathyroidism or hypocalcemia.

All patients were informed in detail regarding the use of $\mathrm{CN}$ and that the cost of the drug would not be reimbursed. The intraoperative use of the drug was according to each patient's own wishes. The technique used is standardized and reported in the literature $[6,12]$. Approval from the Ethics Committee was not necessary.

\section{Tracer}

CNs (LUMMY Pharmaceutical Co., Chongqing, China) were injected as a suspension (50 mg suspended in $1 \mathrm{ml}$ of $0.9 \%$ physiological saline) as the tracer $(0.5 \mathrm{ml}$ per ampoule). The dose for each patient was $0.15 \mathrm{ml}$.

\section{Surgical technique}

The TOET was performed under general anesthesia and in a supine position with neck extension. Following establishment of the working space, a dissection was made at the strap muscles with a Harmonic scalpel, and the strap muscles were pulled laterally with a retractor to exposure the thyroid gland.
The CNs were then injected (Photo $1 \mathrm{~A}$ ). Because of the endoscopic surgery, the CNs could not be injected under direct vision. A percutaneous puncture was made by locating the $\mathrm{CN}$ puncture site on the ceiling skin of the working space. A 22-gauge spinal needle was inserted and passed through the skin, subcutaneous fascia, and platysma until reaching the working space cavity and endoscopic view. The needle was then inserted into the thyroid parenchy$\mathrm{ma}$, and the $\mathrm{CNs}$ were injected into the thyroid gland as a $1.5 \mathrm{ml}$ volume administered to the tumor lobe. After the injection, the syringe was pumped back to avoid injection into blood vessels, while taking care to avoid damage to the thyroid capsule and injection into the tumor. A slow injection speed was needed, and gentle pressure with gauze or absorbable gelatin sponge was applied at the needle puncture site to prevent solution leakage. The lymph vessels and the LNs were stained black. The PGs remained unchanged and were more easily identified and preserved during surgery.

Part of the sternothyroid muscle was transected to expose the upper pole of the thyroid, and the superior PG could easily be seen by lifting the upper pole (Photo $1 \mathrm{~B})$. The recurrent laryngeal nerve, which remained in its original position, was exposed by blunt dissection with endoscopic forceps (Photo $1 \mathrm{C}$ ). Once the inferior PG was found, it was released to the outside. The thyroid gland, including the nodule, was then removed. The CND, which included the prelaryngeal, pretracheal, and paratracheal lymph nodes, was then carried out. The resected LNs in the CN group were carefully checked and labeled as black-staining or unstained (Photo $1 \mathrm{D}$ ). Specimens were also checked for the presence of parathyroid tissue. Once the removal of parathyroid tissue was confirmed, the remainder of the specimen was autotransplanted into the sternocleidomastoid muscle $[13,14]$.

\section{Study design}

All patients underwent preoperative direct laryngoscopy to assess vocal cord motility. $\mathrm{Ca}^{2+}$ and PTH levels were also tested preoperatively. Pathological characteristics (e.g., number of LNs less than $5 \mathrm{~mm}$ removed, total number of LNs removed) and surgery-related indicators (e.g., operative time, blood loss, postoperative drainage, postoperative complications, and in situ preservation of the superior parathyroid and inferior parathyroid) were evaluated. The postoperative follow-up was performed by 

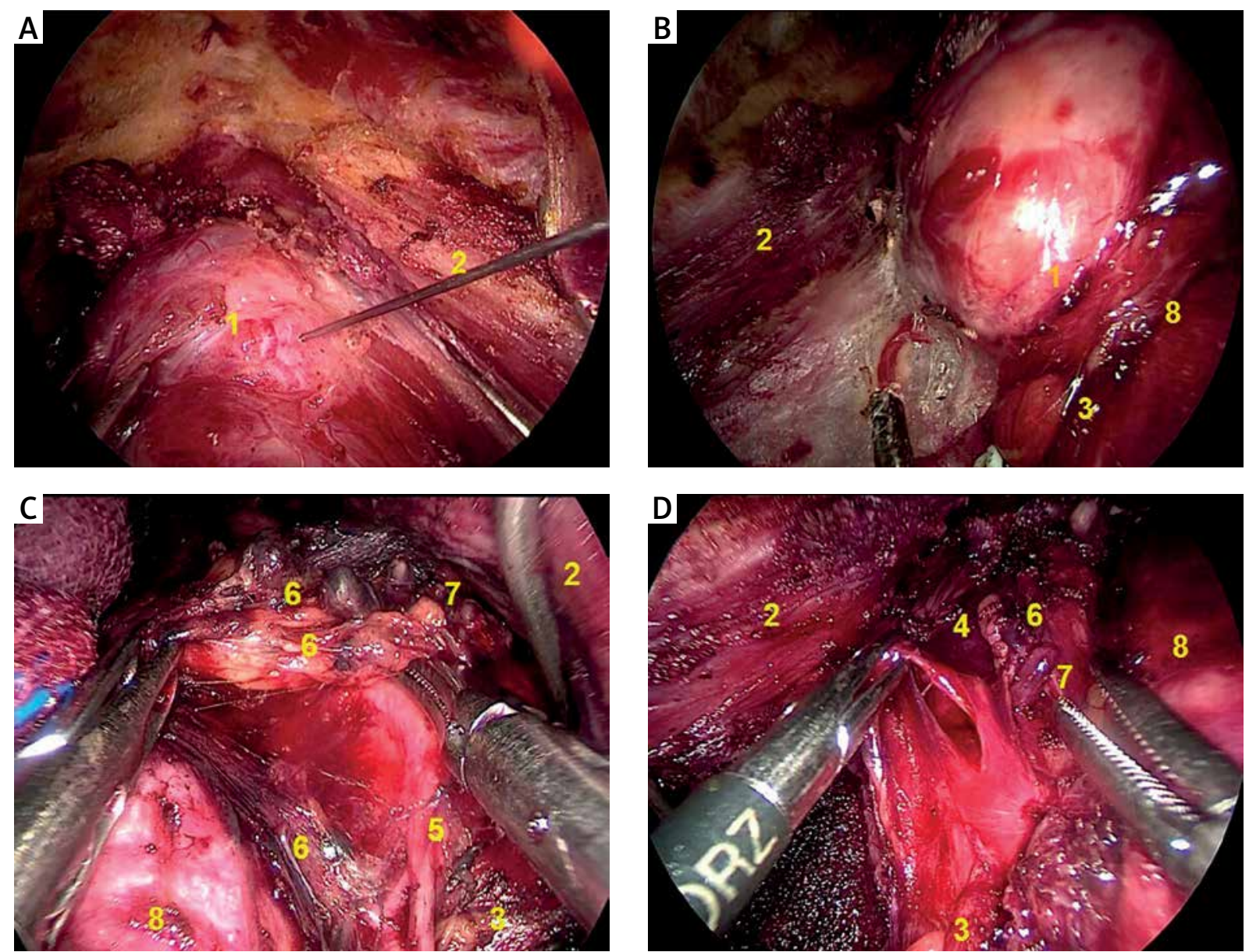

Photo 1. Surgical procedure for transoral vestibular endoscopic thyroidectomy (TOET). 1 - Thyroid gland, 2 - sternocleidomastoid, 3 - superior parathyroid, 4 - inferior parathyroid, 5 - recurrent laryngeal nerve, 6 - black stained lymph node, 7 - unstained lymph node, 8 - trachea

outpatient visits, telephone calls, and written correspondence. Data, including the serum $\mathrm{Ca}^{2+}$ and PTH levels, were gathered at postoperative day 1 , week 1 , month 1 , and month 3 . All patients received calcium gluconate $(2.0 \mathrm{~g} /$ day) for 3 days, regardless of whether they showed a decline in serum or presented signs of hypocalcemia. After 3 days, the patients who still showed a decline in serum $\mathrm{Ca}^{2+}$ and presented signs of hypocalcemia continued taking these drugs.

\section{Statistical analysis}

The $t$-test or Kruskal-Wallis test and $\chi^{2}$ test were used for the continuous and categorical data, respectively. For all statistical analyses, a value of $p<0.05$ was considered statistically significant. All data were processed using SPSS 19.0 software.

\section{Results}

A total of 72 patients ( 68 female and 6 male) were selected over the period from January 2017 to January 2019, and all patients underwent endoscopic unilateral thyroidectomy with CND (level VI) (Figure 1). The clinical characteristics of the patients in both the CN and control groups are summarized in Table I. The patients ranged in age from 19 to 49 years, with a median age of 31 years. No significant differences were found in age, tumor side, sex, primary tumor size, operative time, blood loss, or postoperative drainage (all $p>0.05$ ). No significant differences were noted in operative complications, including neuromuscular symptoms, transient recurrent laryngeal nerve palsy, and swallowing discomfort (all $p>0.05$ ).

The dissected central neck lymph nodes and PGs preserved in situ in the $\mathrm{CN}$ and control groups are 


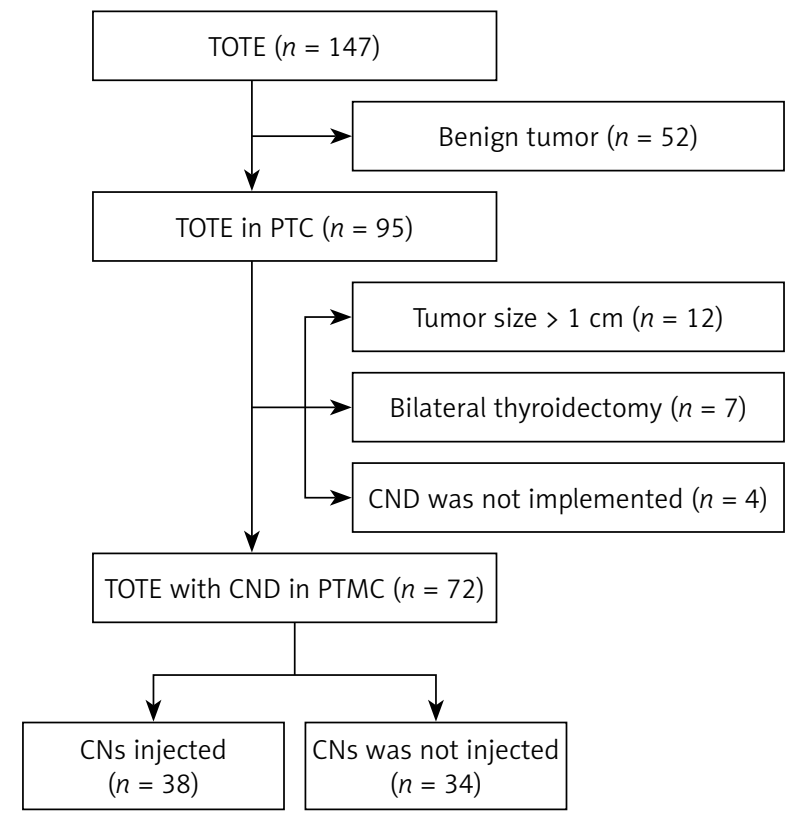

Figure 1. Enrollment chart

compared in Table II. A total of $340 \mathrm{LNs}$ were detected in the $\mathrm{CN}$ group and $233 \mathrm{LNs}$ in the control group. The mean number of detected LNs was significantly higher in the $\mathrm{CN}$ group than in the control group $(8.9 \pm 3.8$ vs. $6.9 \pm 2.7, p=0.021$ ). The resected number of dissected LNs $<5 \mathrm{~mm}$ was greater in the $\mathrm{CN}$ group than in the control group $(3.2 \pm 1.4$ vs. $1.8 \pm 0.8, p<0.01)$.

No significant difference was found in the number of superior PGs preserved in situ (34 vs. 29, $p=0.592$ ), but the number of superior PGs preserved in situ was greater in the CN group than in the control group (29 vs. $18, p=0.038$ ). No significant difference was found in the number of PGs found in the central group of lymph nodes and autotransplanted (6 vs. 8, $p=0.407$ ).

The serum PTH levels dropped markedly in each group on postoperative day 1 and week 1 (Figure 2). However, the PTH levels on postoperative day 1 were similar in both the $\mathrm{CN}$ and the control group (day $1, p=0.946$ ). These levels at postoperative week 1 were significantly lower in the control group than in the $\mathrm{CN}$ group (week 1, $p=0.014$ ). The longterm results revealed similar PTH levels in both the $\mathrm{CN}$ group and the control group at postoperative month 1 and month 3 (month $1, p=0.385$; month 3 , $p=0.827$ ).

The serum $\mathrm{Ca}^{2+}$ levels at each detection point after surgery are shown in Figure 3. The levels on postoperative day 1 and at week 1 were significantly lower in the control group than in the $\mathrm{CN}$ group (day $1, p=0.001$; week $1, p<0.01$ ). The levels at postoperative month 1 and month 3 were similar in both groups (month $1, p=0.829$; month $3, p=0.522$ ).

\section{Discussion}

The development of endoscopic thyroid surgery techniques has led to the wide use of TOET for aesthetically conscious patients with PTMC. Under the magnified and high-resolution vision offered by endoscopy, TOET has advantages over traditional open surgery with respect to central lymph node dissection and protection of the PG blood supply. Our goal in the present study was to determine whether the

Table I. Characteristics and postoperative complications of patients in the $\mathrm{CN}$ and control groups

\begin{tabular}{|lccc|}
\hline Characteristics & $\begin{array}{c}\text { CN group } \\
N=38 \\
n(\%) \text { or mean } \pm \text { SD }\end{array}$ & $\begin{array}{c}\text { Control group } \\
N=34\end{array}$ & $P$-value \\
\hline Age [years] mean \pm SD & $32.6 \pm 7.2$ & 0.217 \\
\hline Gender (male/female) & $30.5 \pm 7.0$ & $3 / 31$ & 0.887 \\
\hline Side (left/right) & $3 / 35$ & $17 / 17$ & 0.824 \\
\hline Tumor size [mm] & $18 / 20$ & $0.69 \pm 0.21$ & 0.559 \\
\hline Operative time [minl] & $0.66 \pm 0.20$ & $106.1 \pm 12.0$ & 0.843 \\
\hline Blood loss [ml] & $106.7 \pm 12.3$ & $21.0 \pm 4.2$ & 0.847 \\
\hline Postoperative drainage [ml] & $21.6 \pm 6.3$ & $50.2 \pm 16.9$ & 0.691 \\
\hline Neuromuscular symptoms & $48.7 \pm 16.5$ & $6(17.6)$ & 0.212 \\
\hline Transient recurrent laryngeal nerve palsy & $3(7.9)$ & $1(2.9)$ & 0.936 \\
\hline Swallowing discomfort & $1(2.6)$ & $2(5.9)$ & 0.909 \\
\hline
\end{tabular}


Table II. Comparison of dissected central neck lymph nodes and parathyroid glands preserved in situ between the $\mathrm{CN}$ group and control group

\begin{tabular}{|lccc|}
\hline Characteristics & $\begin{array}{c}\text { CN group } \\
N=38 \\
n \text { (\%) or mean } \pm \text { SD }\end{array}$ & $\begin{array}{c}\text { Control group } \\
N=34\end{array}$ & $P$-value \\
\hline No. of dissected LNs (mean \pm SD, per patient) & $8.9 \pm 3.8$ & $6.9 \pm 2.7$ & $0.021^{*}$ \\
\hline $\begin{array}{l}\text { No. of dissected } \\
\text { LNs }<5 \text { mm }\end{array}$ & $3.2 \pm 1.4$ & $1.8 \pm 0.8$ & $<0.01^{*}$ \\
\hline Superior parathyroid glands preserved in situ & $34(89.5)$ & $29(85.3)$ & 0.592 \\
\hline $\begin{array}{l}\text { Inferior parathyroid glands preserved in situ } \\
\text { Parathyroid glands found in the central group of lymph } \\
\text { nodes and autotransplanted }\end{array}$ & $6(15.8)$ & $8(23.5)$ & $0.038^{*}$ \\
\hline
\end{tabular}

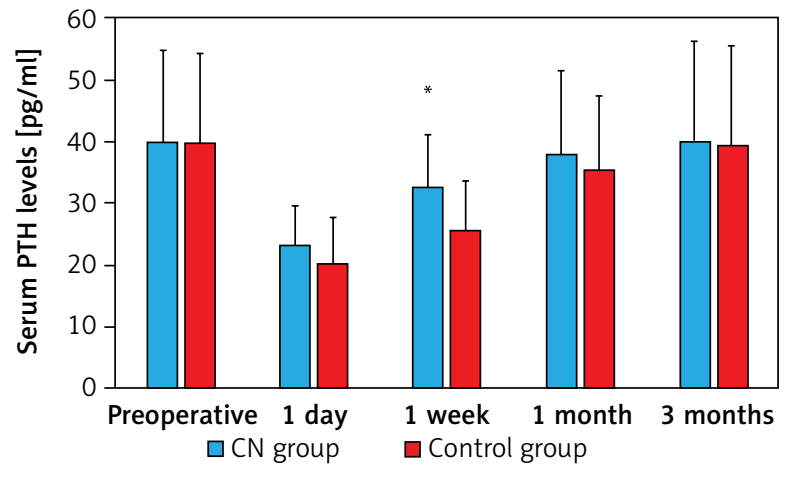

Figure 2. Changes in serum PTH in $\mathrm{CN}$ and the control group after surgery

use of CNs in TOET might further optimize the surgical effects.

The most popular tracer for LNs is methylene blue. However, it has poor specificity, the possibility of postoperative toxic metabolic encephalopathy, and the potential to mask the surrounding tissues and make anatomical structures unclear. Therefore, the utility of methylene blue is not widely accepted by thyroid surgeons. A variety of other techniques, including SPECT images, methoxyisobutylisonitrile scintigraphy using a gamma probe, 5-aminolevulinic acid, and optical coherence tomography, have also been reported but are without clinical effectiveness in terms of protection of the PGs $[15,16]$.

Recently, CN suspensions have been used to aid in the identification of lymph nodes in traditional open thyroid surgery. The product used is a stable suspension of CNs $150 \mathrm{~nm}$ in diameter, a size that ensures that the particles can pass through the lymphatic vessels rather than the blood capillaries. Following injection into the thyroid glands around

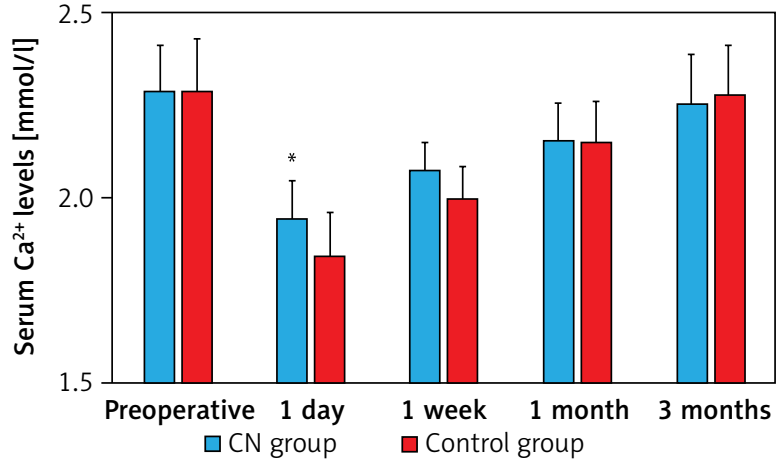

Figure 3. Changes in serum $\mathrm{Ca}^{2+}$ in $\mathrm{CN}$ and the control group after surgery

a thyroid tumor, these CNs are rapidly captured by macrophages. The particles then enter the lymphatic vessels and accumulate in the lymph nodes, causing the thyroid and draining lymph nodes to darken. By contrast, the color of the PG remains unchanged and is more easily identified and preserved during surgery $[17,18]$.

The steps required for injection of the $\mathrm{CN}$ suspension are as follows: (1) the dose of CN suspension should be just $0.15 \mathrm{ml}$ for each lobe, (2) a special needle, long enough for percutaneous injection of the CNs, is needed, and the front end of the needle is bent to help control the depth of the injection, (3) injection into blood vessels and tumors is avoided, (4) an ultrasonic knife is used for closing.

Temporary and permanent hypoparathyroidism occurring after surgery are mainly due to parathyroid contusions or a mistakenly cut or damaged blood supply $[19,20]$. Marked drops in the serum $\mathrm{Ca}^{2+}$ and PTH after surgery imply that PG injury may be inevitable during thyroidectomy with central lymph node 
dissection. The location of the superior parathyroid gland is relatively constant, and no significant difference was found in discovery of the superior parathyroid preserved in situ $(p=0.592)$, Destruction of the inferior PGs may occur more frequently than destruction of the superior PGs because the inferior PGs are adjacent to the inferior pole of the thyroid and the region $\mathrm{VI}$ lymph nodes and because the inferior PGs are often obscured by the thyroid lobe in TOET. However, the use of CNs makes the inferior PGs and lymph nodes easier to identify, and the number of superior PGs preserved discovered in situ was greater in the $\mathrm{CN}$ group than in the control group $(p=0.038)$.

In this study, the number of LNs dissected was higher in the $\mathrm{CN}$ group than in the control group $(p=0.021)$. This may be due to the application of CNs, which enhanced certain smaller LNs or those located in the occult position, and the resected number of dissected LNs $<5 \mathrm{~mm}$ was larger in the CN group than in the control group $(p<0.01)$. However, we did not observe any significant differences in lymph node metastasis rates ( $28.2 \%$ vs. $27.0 \%)$ between the two groups. Our results also revealed no differences in the number of metastatic lymph nodes between the black-stained and unstained lymph nodes ( $28.8 \%$ vs. $26.5 \%)$, suggesting that the distribution of the $\mathrm{CN}$ lymph node tracer was not associated with lymph node metastasis.

The patients in both groups showed significantly lower serum $\mathrm{Ca}^{2+}$ and PTH levels than the preoperative levels at postoperative day 1 and week 1 , and the changes in PTH levels were closely related to those of the $\mathrm{Ca}^{2+}$ levels. We found that patients who received the $\mathrm{CN}$ injection demonstrated small changes in the trends of PTH and $\mathrm{Ca}^{2+}$ levels postoperatively following surgery. The parathyroid function was also restored in a shorter time in the $\mathrm{CN}$ group.

This study had some limitations. Selection bias may have existed regarding which procedure was chosen, as this study is a retrospective study and not a blind randomized study. The follow-up period was also very short. The effect of $\mathrm{CN}$ use on the long-term outcomes of TOET is also unclear, and the sample size was too small.

\section{Conclusions}

The clinical utilization of a $\mathrm{CN}$ suspension in TOET can provide a more accurate identification of LNs, thereby increasing the number of lymph nodes retrieved and playing a role in protecting parathyroid function.

\section{Conflict of interest}

The authors declare no conflict of interest.

\section{References}

1. La Vecchia C, Malvezzi M, Bosetti C, et al. Thyroid cancer mortality and incidence: a global overview. Int J Cancer 2015; 136 : 2187-95.

2. Davies L, Welch HG. Current thyroid cancer trends in the United States. JAMA Otolaryngol Head Neck Surg 2014; 140: 317-22.

3. Siegel RL, Miller KD, Jemal A. Cancer statistics, 2019. CA Cancer J Clin 2019; 69: 7-34.

4. Macedo FI, Mittal VK. Total thyroidectomy versus lobectomy as initial operation for small unilateral papillary thyroid carcinoma: a meta-analysis. Surg Oncol 2015; 24: 117-22.

5. Donatini G, Castagnet M, Desurmont T, et al. Partial thyroidectomy for papillary thyroid microcarcinoma:is completion total thyroidectomy indicated. World J Surg 2016; 40: 510-5.

6. Yang J, Wang C, Li J, et al. Complete endoscopic thyroidectomy via oral vestibular approach versus areola approach for treatment of thyroid Diseases. J Laparoendosc Adv Surg Tech A 2015; 25: 470-6.

7. Dionigi G, Tufano RP, Russell J, et al. Transoral thyroidectomy: advantages and limitations. J Endocrinol Invest 2017; 40: 1259-63.

8. Pattou F, Combemale F, Fabre S, et al. Hypocalcemia following thyroid surgery: incidence and prediction of outcome. World J Surg 1998; 22: 718-24.

9. Hillary SL, Guillermet S, Brown NJ, et al. Use of methylene blue and near-infrared fluorescence in thyroid and parathyroid surgery. Langenbecks Arch Surg 2018; 403: 111-8.

10. Anuwong A, Ketwong K, Jitpratoom P, et al. Safety and outcomes of the transoral endoscopic thyroidectomy vestibular approach. JAMA Surg 2018; 153: 21-7.

11. Yu W, Cao X, Xu G, et al. Potential role for carbon nanoparticles to guide central neck dissection in patients with papillary thyroid cancer. Surgery 2016; 160: 755-61.

12. Zhu Y, Chen $X$, Zhang H, et al. Carbon nanoparticle-guided central lymph node dissection in clinically node-negative patients with papillary thyroid carcinoma. Head Neck 2016; 38: 840-5.

13. Wang B, Qiu NC, Zhang W, et al. The role of carbon nanoparticles in identifying lymph nodes and preserving parathyroid in total endoscopic surgery of thyroid carcinoma. Surg Endosc 2015; 29: 2914-20.

14. Kim WW, Lee J, Jung JH, et al. A comparison study of the transoral and bilateral axillo-breast approaches in robotic thyroidectomy. J Surg Oncol 2018; 118: 381-7.

15. Hara N, Takayama T, Onoguchi M, et al. Subtraction SPECT for parathyroid scintigraphy based on maximization of mutual information. J Nucl Med Technol 2007; 35: 84-90.

16. Patel HP, Chadwick DR, Harrison BJ, et al. Systematic review of intravenous methylene blue in parathyroid surgery. Br J Surg 2012; 99: 1345-51. 
17. Li Y, Jian WH, Guo ZM, et al. A meta-analysis of carbon nanoparticles for identifying lymph nodes and protecting parathyroid glands during surgery. Otolaryngol Head Neck Surg 2015; 152: 1007-16.

18. Sun SP, Zhang Y, Cui ZQ, et al. Clinical application of carbon nanoparticle lymph node tracer in the VI region lymph node dissection of differentiated thyroid cancer. Genet Mol Res 2014; 13: 3432-7.

19. Roh JL, Park JY, Park Cl. Total thyroidectomy plus neck dissection in differentiated papillary thyroid carcinoma patients: pattern of nodal metastasis, morbidity, recurrence, and postoperative levels of serum parathyroid hormone. Ann Surg 2007; 245 604-10.

20. Giordano D, Valcavi R, Thompson GB, et al. Complications of central neck dissection in patients with papillary thyroid carcinoma: results of a study on 1087 patients and review of the literature. Thyroid 2012; 22: 911-7.

Received: 2.08 .2019 , accepted: 17.10 .2019 